Article

\title{
Current-Fluctuation Mechanism of Field Emitters Using Metallic Single-Walled Carbon Nanotubes with High Crystallinity
}

\author{
Norihiro Shimoi * (D) and Kazuyuki Tohji \\ Graduate School of Environmental Studies, Tohoku University, 6-6-20 Aoba, Aramaki, Aoba-ku, \\ Sendai 980-8579, Japan; kazuyuki.tohji.a8@tohoku.ac.jp \\ * Correspondence: norihiro.shimoi.c8@tohoku.ac.jp; Tel.: +81-22-795-4584
}

Received: 17 November 2017; Accepted: 14 December 2017; Published: 19 December 2017

\begin{abstract}
Field emitters can be used as a cathode electrode in a cathodoluminescence device, and single-walled carbon nanotubes (SWCNTs) that are synthesized by arc discharge are expected to exhibit good field emission (FE) properties. However, a cathodoluminescence device that uses field emitters radiates rays whose intensity considerably fluctuates at a low frequency, and the radiant fluctuation is caused by FE current fluctuation. To solve this problem, is very important to obtain a stable output for field emitters in a cathodoluminescence device. The authors consider that the electron-emission fluctuation is caused by Fowler-Nordheim electron tunneling and that the electrons in the Fowler-Nordheim regime pass through an inelastic potential barrier. We attempted to develop a theoretical model to analyze the power spectrum of the FE current fluctuation using metallic SWCNTs as field emitters, owing to their electrical conductivity by determining their FE properties. Field emitters that use metallic SWCNTs with high crystallinity were successfully developed to achieve a fluctuating FE current from field emitters at a low frequency by employing inelastic electron tunneling. This paper is the first report of the successful development of an inelastic-electron-tunneling model with a Wentzel-Kramers-Brillouin approximation for metallic SWCNTs based on the evaluation of FE properties.
\end{abstract}

Keywords: field emission; metallic single-walled carbon nanotube; high crystallinity; current fluctuation; inelastic electron tunneling; Fowler-Nordheim tunneling

\section{Introduction}

Field emitters can be used as cathodoluminescence (CL) devices, which radiate rays with a wide range of frequencies from $X$-ray to far infrared rays. However, they have a serious problem in that the field emission (FE) current depends on the degree of vacuum, owing to the adhesion of contaminants such as residual gas or water on the field emitter surface [1,2]. The emitter surface needs to be clean because the FE and carbon nanotubes (CNTs) are expected to enable field emitters to realize stable emission. However, the emission current from field emitters, including CNTs, has been reported to fluctuate even in a high-vacuum atmosphere [3,4]. A CL device that uses a field emitter as a cathode electrode radiates rays that considerably fluctuate or flicker. The fluctuation or flicker from a CL device mainly has low frequencies of under $200 \mathrm{~Hz}$ [5-10]. On the basis of the above problems, a cathode structure that can reduce the fluctuation of emission has been studied $[9,10]$. However, a technique to reduce the fluctuation is not yet well established. In the case of FE, the electrons that are emitted from field emitters probabilistically and quantum-mechanically pass through a quantum barrier with a thickness of nm order before they are emitted in vacuum. This distance is estimated from the thickness of the electron-tunneling barrier when electrons cause Fowler-Nordheim tunneling [11,12]. In the case 
of Fowler-Nordheim tunneling, electrons have been considered to inelastically pass through a barrier, and the electrons that are emitted from an FE cathode exhibit energy fluctuation.

Therefore, we have carried out basic research to develop FE devices using single-walled CNTs (SWCNTs), and, for the first time, succeeded in employing SWCNT field emitters as the cathode of a planar lighting device with good emission stability at a high current density of over $10 \mathrm{~mA} / \mathrm{cm}^{2}[13,14]$. Since CNTs were first reported by Iijima [15], it has been shown that they exhibit one-dimensional confinement effects and can be used as coherent quantum wires [16-18]. Among the CNTs, the Young's modulus of SWCNTs is particularly high [19]. SWCNTs have been used in a wide range of applications, including field emitters, probes in scanning microscopes [20], gas-storage materials [21], and electrode materials for secondary batteries [22], and have been studied in a wide range of applied research fields. In recent studies of applications using CNTs, the development of electrical stability as field emitters requires the fabrication of efficient CL devices, such as flat panel displays with high brightness efficiency and low power consumption [23].

We considered that an FE cathode comprising well-dispersed SWCNTs in a high-vacuum atmosphere is indispensable for decreasing the current fluctuation of field emitters and for improving the homogeneity of planar emission from a planar lighting device [13]. However, we consider that the FE fluctuation cannot be reduced to zero. In this study, to demonstrate the above consideration, the fluctuation effect on the properties of field emitters that use metallic SWCNTs with high purity and crystallinity was examined. We developed field emitters using these SWCNTs, and theoretically analyzed the fluctuation of their power spectrum in terms of their electrical conductivity by determining their electrical properties (FE properties).

\section{Experimental}

A wet coating process was adopted to fabricate a cathode with stable $\mathrm{FE}$ at a low driving voltage using metallic SWCNTs (NanoIntegris Technologies Inc., Boisbriand, QC, Canada). The homogeneous dispersion of the SWCNTs in a liquid medium is the key factor for obtaining a planer electron emission homogeneously as a field emitter. Highly crystalline metallic SWCNTs are expected to lower the threshold field and stably emit electrons at a low driving voltage. However, it is difficult to homogeneously disperse highly crystalline SWCNTs in liquids [24]. In general, a dispersant is used to facilitate the homogeneous dispersion of nanocarbon materials in liquids. However, highly crystalline SWCNTs cannot bind to the functional groups in a dispersant because there are very few defective binding sites in their carbon network that can be used for binding to the functional groups in the dispersant. We attempted to physically separate highly crystalline SWCNT aggregates into uniform single nanotubes in a low-viscosity solvent, containing $\mathrm{In}_{2} \mathrm{O}_{3}-\mathrm{SnO}_{2}$ [tin-doped indium oxide (ITO)] precursor solution. A non-ionic dispersant was added to the solvent to facilitate the dispersion of individual SWCNTs, and the mixture was agitated using a homogenizer.

To prepare the coating film, butyl acetate (99\%) and ethyl cellulose (EC) ( 49\%, ethoxy $100 \mathrm{cP})$, which were obtained from Wako Pure Chemical Industries, Ltd., Osaka, Japan, and an ITO precursor solution (Kojundo Chemical Laboratory Co., Ltd., Sakado, Japan) were used. The initial mixture was prepared by mixing metallic SWCNTs, which were purified from solutions from NanoIntegris Technologies Inc., the ITO precursor solution, butyl acetate added as required to control the viscosity of the solution, and EC as a surfactant at a weight ratio of 1:8:600:10. Then, the mixture was agitated using the homogenizer for $30 \mathrm{~min}$ at room temperature. The agitated mixture was sprayed on a silicon substrate to form a film. The film was dried at approximately $420 \mathrm{~K}$ and sintered at $720 \mathrm{~K}$ in vacuum to remove any organic-solvent components. As a result, ITO films were obtained from each type SWCNT, which were used as cathode plates. In addition, to facilitate field emission, the sintered ITO film with dispersed SWCNTs was scratched to activate the film surface and obtain stable FE properties [25].

Figure 1 shows (a) a schematic diagram of SWCNTs that were protruding from a scratched groove, (b) a scanning electron microscopy (SEM, SU-8000, Hitachi High-Technologies Corporation, Tokyo, Japan) overview after activating the FE for the ITO film, including metallic SWCNTs, and (c) an 
enlarged view of the protruding SWCNTs in the circled region of (b). After the film that was coated with the mixture was sintered in vacuum, it was activated by scratching using a thin metal rod to obtain the FE properties at a low turn-on field. We observed that the bundles of SWCNTs protruded from both sides of the edge of the scratched ITO film. The exposed SWCNTs in the ITO film were homogeneously dispersed and were oriented in random directions. Furthermore, the SWCNTs that were used for an FE thin film were checked for high crystallization by transmission electron microscope (TEM) (HR-3000, Hitachi High-Tech. Co. Ltd., Tokyo, Japan) measurement in Figure 1d. Each SWCNT in a bundle of Figure $1 \mathrm{~d}$ exhibits a clear TEM image of each tube wall identified as straight lines. The TEM image in Figure 1d explains that a SWCNT has highly crystalline carbon network. Namely, we can expect to develop field emitters employing these SWCNTs, and theoretically analyze the effect of the increased crystallinity of SWCNTs on their electrical conductivity by determining their electrical properties (FE properties).
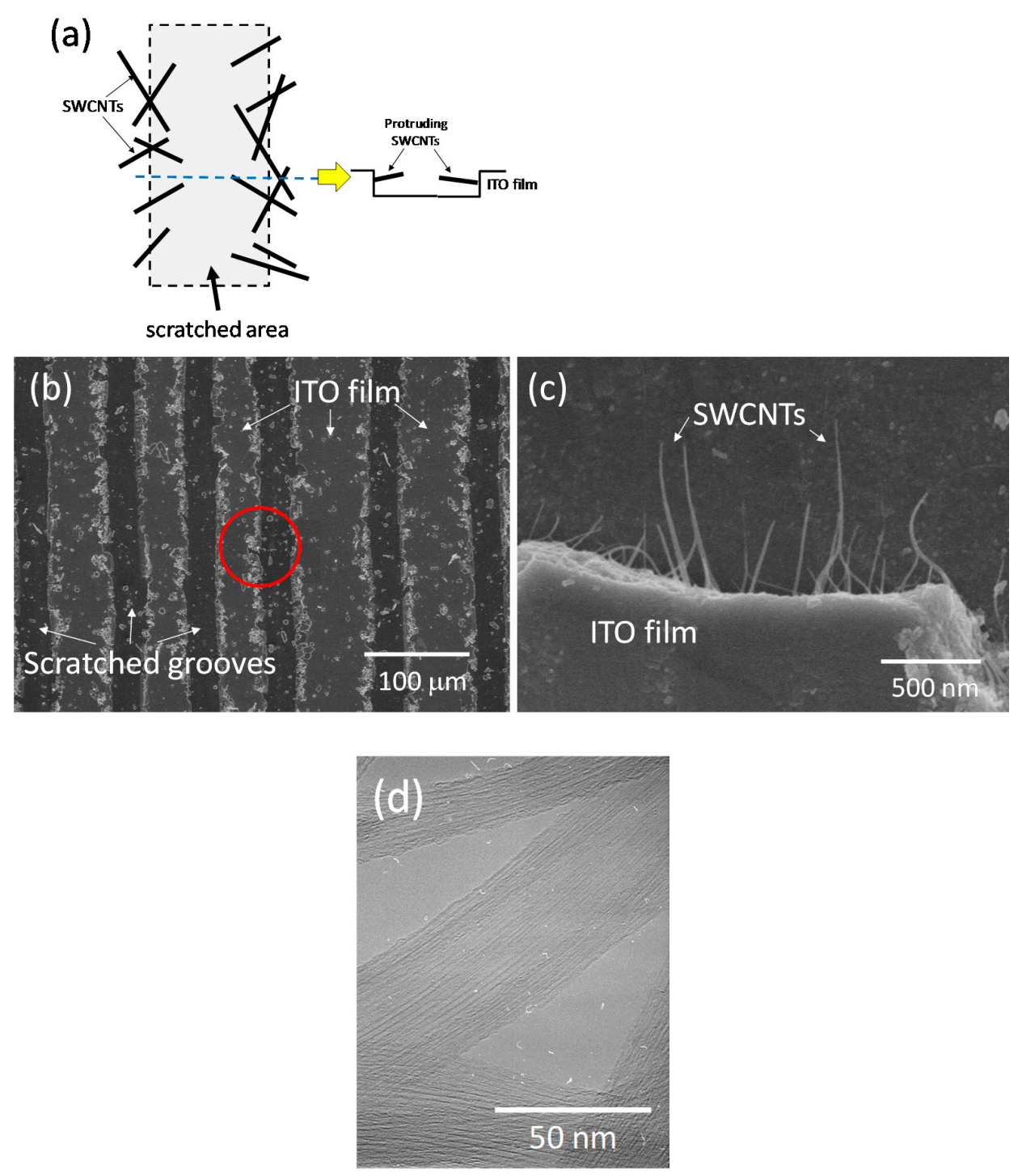

Figure 1. Tin-doped indium oxide (ITO) film images after field emission (FE) activation. (a) Schematic diagram of the scratched area and single-walled carbon nanotubes (SWCNTs) protruding from the ITO film; (b) Scanning electron microscope (SEM) overview after scratching the ITO film, including metallic SWCNTs to activate FE; and, (c) Enlarged SEM view of the metallic SWCNTs protruding from the ITO film; (d) Transmission electron microscope (TEM) overview of bundles of metallic highly crystalline SWCNTs that were used in the ITO film $(\mathbf{b}, \mathbf{c})$. 
A diode structure with a fixed gap $(1.2 \mathrm{~mm})$ between the ITO film (cathode) and a phosphor plate coated on ITO electrode (anode) was adopted for the FE measurement. Figure 2 shows a schematic of the system used for FE measurement.

The obtained sample was placed in a vacuum chamber system and maintained at a pressure of $10^{-6} \mathrm{~Pa}$. Direct current (DC) voltage was applied across the cathode and anode to determine the FE properties after baking the sample to reduce the amount of gas contaminating the SWCNT surface. A power supply unit and a PC were connected to automatically store data.

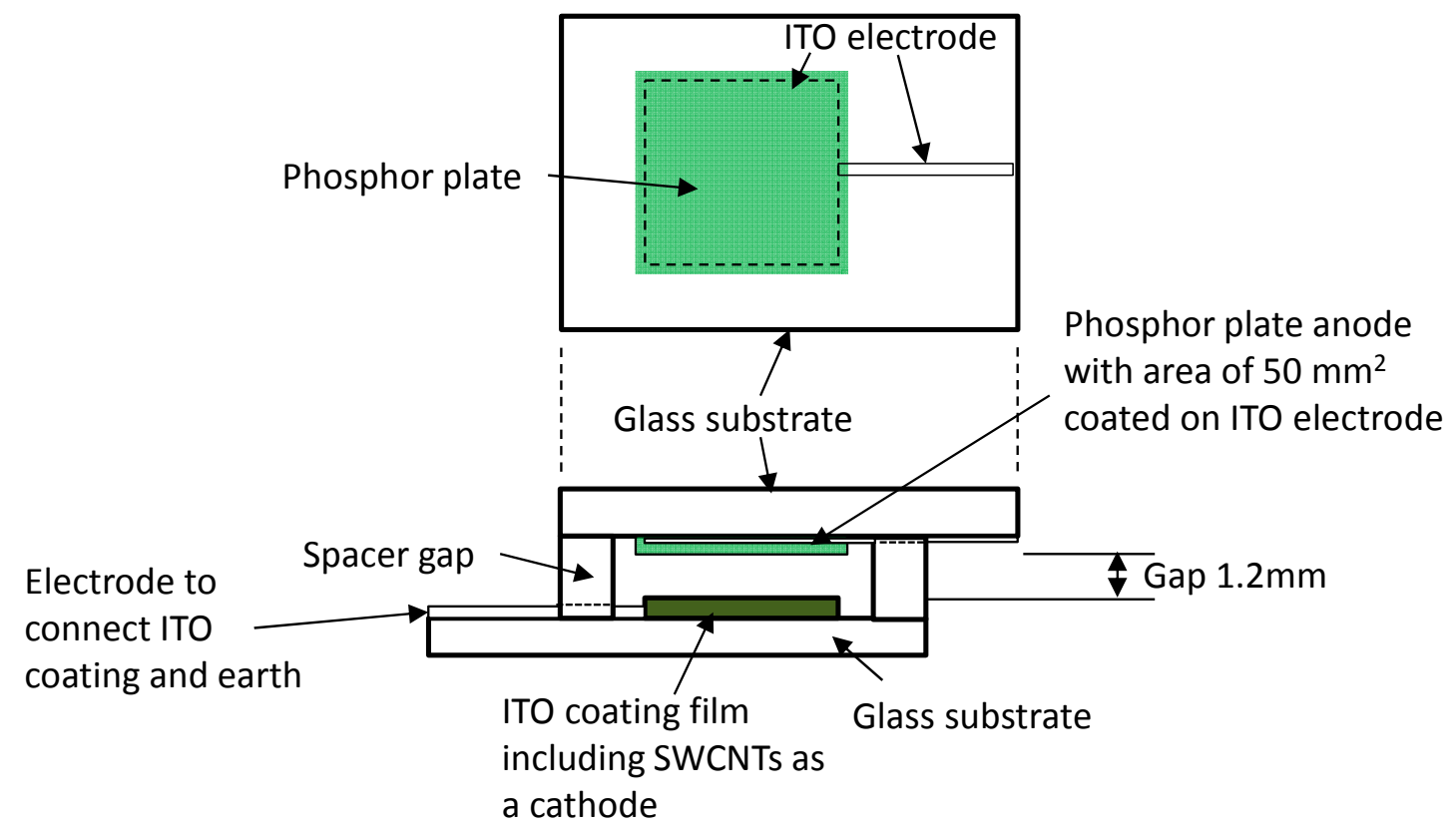

Figure 2. Schematic diagram of the FE measurement system with a diode structure.

\section{Theory of FE Fluctuation}

The field emission tunneling model was originally represented by the electron tunneling model, as expressed by the Spindt model based on the Fowler and Nordheim (F-N) model [26]. The model has the basis of the electron tunneling with ballistic electrons considered to pass through the energy barrier from SWCNTs, however it is valid only when the flow of electrons in the field emitter is prevented by an energy barrier. In the FE model used in this study, we assumed that electrons are emitted from SWCNTs to the outside, and we constructed an FE model by combining the model of inelastic tunneling electrons passing through SWCNTs into the F-N tunneling model of electrons emitted to the outside.

In the inelastic-electron-tunneling model, the states of the electrons before and after tunneling through an energy barrier are estimated by considering the energy transfer. According to Laks and Mills [27], $\hat{J}(\vec{x}, t)$ in the current-current correlation $\left\langle\hat{J}(\vec{x}, t)^{*} \hat{J}(\vec{x}, t)\right\rangle$ can be expressed in the following form:

$$
\hat{I}(t)=\int d x_{/ /} \hat{J}(\vec{x}, t)
$$

$\hat{I}(t)$ is the quantum-mechanical tunneling-current operator and represents the current flow at time $t$. The normalization $P$ of the wave function $\psi(\vec{x}, t)$ is expressed as the probability density of the existence of electrons in a quantum barrier, as follows:

$$
P(\vec{x}, t)=|\psi(\vec{x}, t)|^{2}=\psi^{*}(\vec{x}, t) \psi(\vec{x}, t)
$$


when an electron exists in sphere $\Omega$

$$
\begin{aligned}
& \frac{\delta}{\delta t}\left(\int_{\Omega} P(\vec{x}, t) d \vec{x}\right)=\int_{\Omega}\left(\psi^{*} \frac{\delta \psi}{\delta t}\right) d \vec{x} \\
& \quad=\frac{1}{i \hbar} \int_{\Omega}\left(\psi^{*} H \psi-\psi H \psi^{*}\right) d \vec{x} \\
& \quad=\frac{i \hbar}{2 m} \int_{A}\left(\psi^{*} \nabla \psi-\psi \nabla \psi^{*}\right) d \vec{A}
\end{aligned}
$$

when $S(\vec{x}, t) \equiv \frac{\hbar}{2 i m}\left(\psi^{*} \nabla \psi-\psi \nabla \psi^{*}\right)$,

$$
\frac{\partial}{\partial t} \int_{\Omega} P(\vec{x}, t) d \vec{x}=-\int_{\Omega} \nabla S(\vec{x}, t) d \vec{x}
$$

when $\frac{\partial}{\partial t} P(\vec{x}, t)+\nabla \mathrm{S}(\vec{x}, t)=0$,

$$
\begin{gathered}
\hat{J}(\vec{x}, t)=-e \hat{S}(\vec{x}, t) \\
=\frac{i e \hbar}{2 m}\left(\psi^{*} \nabla \psi-\psi \nabla \psi^{*}\right)
\end{gathered}
$$

where $e$ is the electric charge and $\hat{I}(t)$ is expressed as

$$
\hat{I}(t)=\sum_{k \cdot q}\left[T_{k q} C_{q}^{r+}(t) C_{k}^{l}(t)+T_{k q}^{*} C_{\Re}^{l+}(t) C_{q}^{r}(t)\right],
$$

where $T_{k q}$ is the tunneling matrix element between state $k$ in the source electrode and state $q$ in the vacuum through the tunneling barrier. $C_{q}^{r+}(t)$ and $C_{R}^{l}(t)$ are the creation and annihilation operators of the electron, respectively. Using the Wentzel-Kramers-Brillouin (WKB) method to calculate the electron wave functions, the tunneling matrix element can be expressed as [28-30]

$$
\left|T_{\varkappa_{q}}\right| \propto \frac{\hbar^{2}}{2 m^{*}} \exp \left(-\int_{z_{l}}^{z_{r}} d z\left|\hbar_{z}(z)\right|\right) \exp \left(-\int_{z_{l}}^{z_{r}} d z\left|q_{z}(z)\right|\right)
$$

where $m^{*}$ is the effective mass of an electron in the tunneling barrier, and $k_{\approx}$ and $q_{z}$ are the components of the electron wave vector in the barrier that is perpendicular to the CNT surface.

$$
\begin{gathered}
\hbar_{z}(z)=\frac{\sqrt{2 m^{*}}}{\hbar}\left[V(z)-E+\frac{\hbar^{2}}{2 m^{*}} \hbar^{2}\right]^{\frac{1}{2}} \\
q_{z}(z)=\frac{\sqrt{2 m^{*}}}{\hbar}\left[V(z)-E+\frac{\hbar^{2}}{2 m^{*}} \hbar^{2}+\hbar \omega\right]^{\frac{1}{2}} .
\end{gathered}
$$

here, $V(z)$ is the barrier potential and $z$ is the coordinate of the axis perpendicular to the interfaces. The average of the current-current correlation in state $i$ is expressed as:

$$
\left\langle\hat{I}^{*}(t) \hat{I}(0)\right\rangle=\frac{1}{N} \sum_{i}\left\langle i\left|\hat{I}^{*}(t) \hat{I}(0)\right| i\right\rangle .
$$

$|I(\omega)|^{2}$ is the power spectrum of the fluctuation of the tunneling current for the FE mechanism.

$$
|I(\omega)|^{2}=\int_{-\infty}^{\infty}\left\langle\hat{I}^{*}(t) \hat{I}(0)\right\rangle e^{-i \omega t} d t
$$

By substituting Equations (6)-(9) into Equation (11), we finally obtain the following expression for the power spectrum of the FE current fluctuation: 


$$
|I(\omega)|^{2}=\frac{e^{2} A m}{2 \pi^{2} \hbar^{3}} \int d E \times \exp \left(-\int d z\left|\hbar_{z}(z)\right|\right) \exp \left(-\int d z\left|q_{z}(z)\right|\right) .
$$

where $A$ is corresponding to the total area of the FE site, which electrons emit from the SWCNTs the cathode and $m$ is the effective mass of an electron that moves parallel to the emission site in the source electrode, from which tunneling electrons are injected into the barrier.

Equation (12) should be transformed into a more suitable form for an FE cathode using metallic SWCNTs. FE model was carried out in the Fowler-Nordheim tunneling regime with the barrier potential $V(z)$, and a simplified form for the power spectrum is expressed as:

$$
|I(\omega)|^{2} \propto \frac{e^{3} A}{4 \pi^{2} \hbar \phi_{B}} \cdot|E|^{2}\left(\frac{\hbar \omega}{e \phi_{B}}+1\right)^{-1} \times \exp \left\{-\frac{2 \sqrt{2 m}}{3 \hbar e|E|}\left((\hbar \omega)^{\frac{3}{2}}+\left(e \phi_{B}\right)^{\frac{3}{2}}\right)\right\} .
$$

here, $\phi_{B}$, as shown in Figure 3 is the barrier height potential, which is the difference from the vacuum level to the energy level of the bottom of the Fermi level in the case of metallic SWCNTs, and $E$ is the field applied to the SWCNTs, which is enhanced by the tip shape. The enhancement factor $\beta$ of $E$ is calculated by simulation using the surface-charge method with a three-dimensional model of SWCNTs protruding from the ITO film [31-33], where:

$$
E=\beta E_{0}
$$

there, $E_{0}$ is the actual electrical field supplied between the cathode and anode electrodes. Moreover, field emission site $A$ on the SWCNTs of the cathode was estimated from the comparison between the simulated and measured bright spots [31].

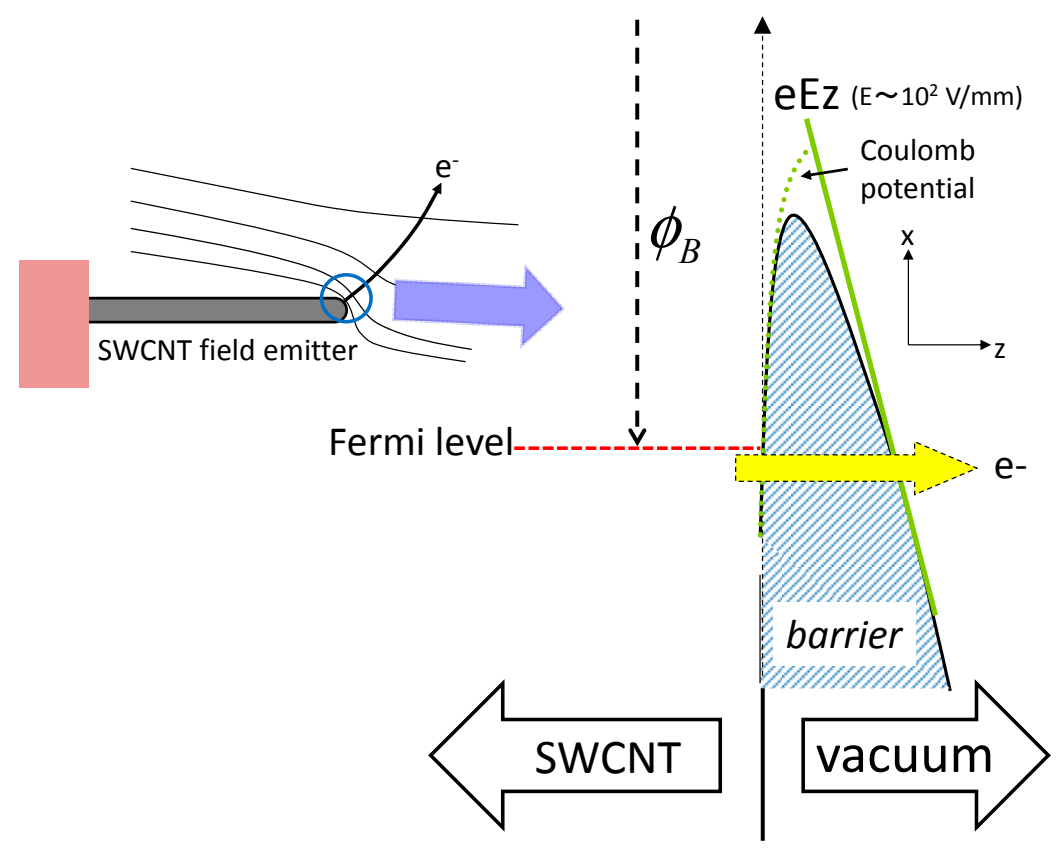

Figure 3. Primitive model of the FE using an SWCNT emitter based on Fowler-Nordheim tunneling.

\section{Results and Discussion}

Figure 4 shows the relationship between the current density and electric field of the SWCNTs that were obtained using the FE measurement system (Figure 2). The threshold field of the metallic SWCNTs was $4.1 \mathrm{~V} / \mu \mathrm{m}$ at a line-current density of $0.1 \mathrm{~mA} / \mathrm{cm}^{2}$, as shown in Figure 4 . Moreover, the planar lighting at $2 \mathrm{~mA} / \mathrm{cm}^{2}$ was shown in the inset of Figure 4. 


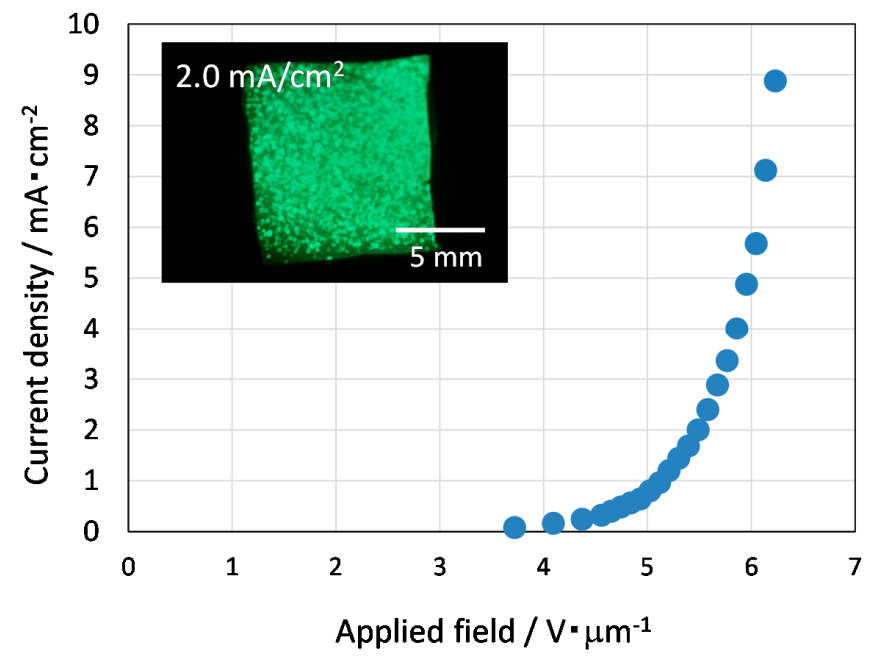

Figure 4. Current density-electric-field characteristics of metallic SWCNTs. The inset shows the planar lighting.

Figure 5 shows the FE current density of metallic SWCNTs with different supplied DC voltages over $40 \mathrm{~s} \mathrm{(a)}$ and the distribution of the power spectrum of the frequency based on information on the FE current fluctuation, as obtained by the Fourier transform method (b). The sample was biased using a DC power supply, and the current density from the DC power supply was measured to be $1.2 \mathrm{~mA} / \mathrm{cm}^{2}$ at $5.2 \mathrm{~V} / \mu \mathrm{m}(6 \mathrm{kV}), 2.0 \mathrm{~mA} / \mathrm{cm}^{2}$ at $5.5 \mathrm{~V} / \mu \mathrm{m}(6.6 \mathrm{kV}), 4.6 \mathrm{~mA} / \mathrm{cm}^{2}$ at $5.9 \mathrm{~V} / \mu \mathrm{m}(7.1 \mathrm{kV})$, and $10 \mathrm{~mA} / \mathrm{cm}^{2}$ at $6.3 \mathrm{~V} / \mu \mathrm{m}(7.6 \mathrm{kV})$. We observed a high stability of the FE current, owing to the desorption of gases on the SWCNTs that was caused by the annealing before the FE measurement. We would have observed some damped current distributions in the FE current if the annealing of the sample had been insufficient. However, Figure 5a shows no damping, and the SWCNT surfaces were considered to have been sufficiently cleaned before the FE measurement. The spectrum of each FE current, as shown in Figure 5a, is normalized by the peak at $0 \mathrm{~Hz}$, and indicates that periodic current fluctuation is present in the FE. A peak exists at $50 \mathrm{~Hz}$, as shown in Figure $5 \mathrm{~b}$, originating from the noise of the DC power supply, which corresponds to the frequency of the commercial power supply in the east of Japan. We observed that the stable DC current in the FE images obtained from the power spectrum, as shown in Figure 5a, has an alternating-current component, particularly at low frequencies of under approximately $100 \mathrm{~Hz}$. Moreover, the spectrum distribution depends on the magnitude of the FE current, and the alternating-current component of each spectrum increases with the DC power.

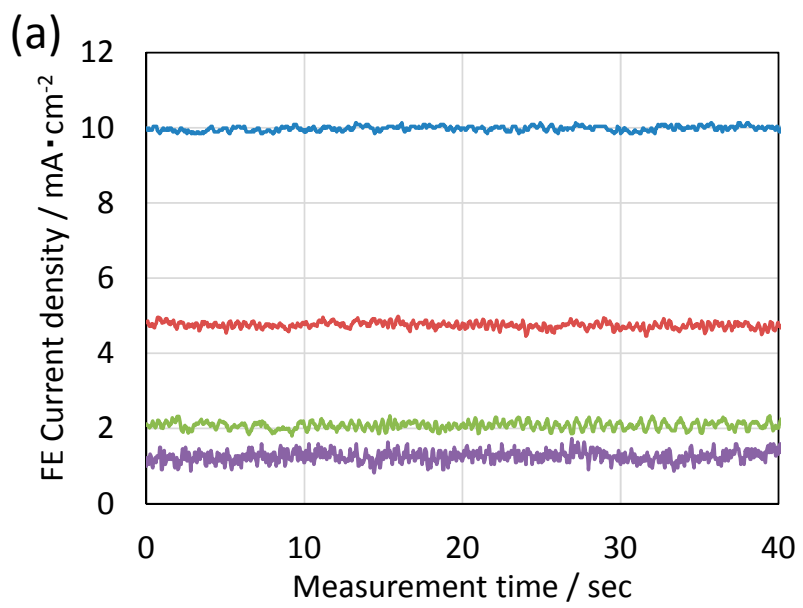

Figure 5. Cont. 
(b)

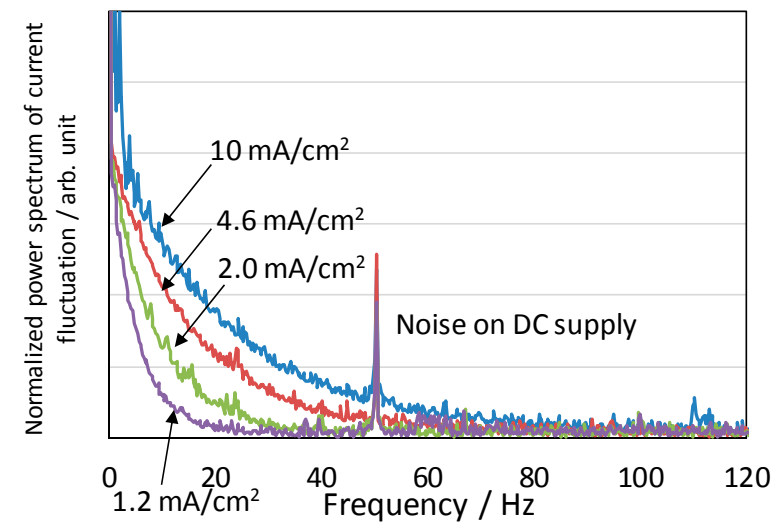

Figure 5. Distributions of (a) FE current fluctuation and (b) spectrum of the FE current fluctuation obtained by the Fourier transform method.

We compared the above experimental results with the theoretical predictions described in Section 2. The parameters that determine the tunneling characteristics of the field emitters that used metallic SWCNTs (shown in Figures 4 and 5) are listed in Table 1. The enhanced field $E$ in Equation (13) was determined by simulation using the surface electrical method used in Ref. [30], with the SWCNT model. The emission site $A$ at the SWCNT surface was estimated and calculated from the comparison between the simulated and measured bright spots [31]. $\phi_{B}$ was estimated from the work function of bulk carbon.

Table 1. Parameters used in the theoretical calculations. $A$ and $\beta$ were obtained by simulation using the method in Ref. [29,30].

\begin{tabular}{cc}
\hline$e$ (elementary charge) & $1.60 \times 10^{-19} \mathrm{C}$ \\
\hline$A$ (FE site) & $6.48 \times 10^{-24} \mathrm{~m}^{2}$ \\
$\phi_{\mathrm{B}}$ (work function of bulk carbon) & $4.3 \mathrm{eV}$ \\
$\hbar(=h / 2 \pi, h$ : Planck constant) & $6.58 \times 10^{-16} \mathrm{eV} \cdot \mathrm{s}$ \\
$\beta$ (enhancement factor) & $2.56 \times 10^{3}$ \\
$m$ (free electron mass) & $9.11 \times 10^{-31} \mathrm{~kg}$ \\
\hline
\end{tabular}

The power spectrum $|I(\omega)|^{2}$ obtained from Equation (13), which is based on the WKB approximation, and Table 1 show the dependence on the voltage applied between a cathode with SWCNTs and an anode electrode, as shown in Figure 6. Each spectrum distribution depends on the magnitude of the FE current, and the alternating-current component of each spectrum increases with the DC power. For the metallic SWCNTs, the experimentally obtained results are shown in Figure 5, and those that are obtained by simulation are shown in Figure 6, which are in good agreement. Distribution of the energy of electrons that pass through an energy barrier by WKB approximation expands when an applied voltage between a cathode and an anode electrode increases. Namely, this means that the high frequency components of FE current increase, and it is guessed that high frequency components of the power spectrum relatively increase by increasing in an applied voltage. On the basis of this agreement, the experimental results were modeled by the inelastic tunneling model based on the Fowler-Nordheim tunneling of electrons emitted from the ends of the SWCNTs using the current-fluctuation power spectrum given by Equation (13). The results that are shown in Figures 5 and 6 indicate that an electron passing into a barrier in the Fowler-Nordheim regime constantly exhibit an electrical-charge fluctuation in the inelastic tunneling model, and the shape of the power spectrum depends on the quantity of electrical charge that is emitted from the field emitters. 


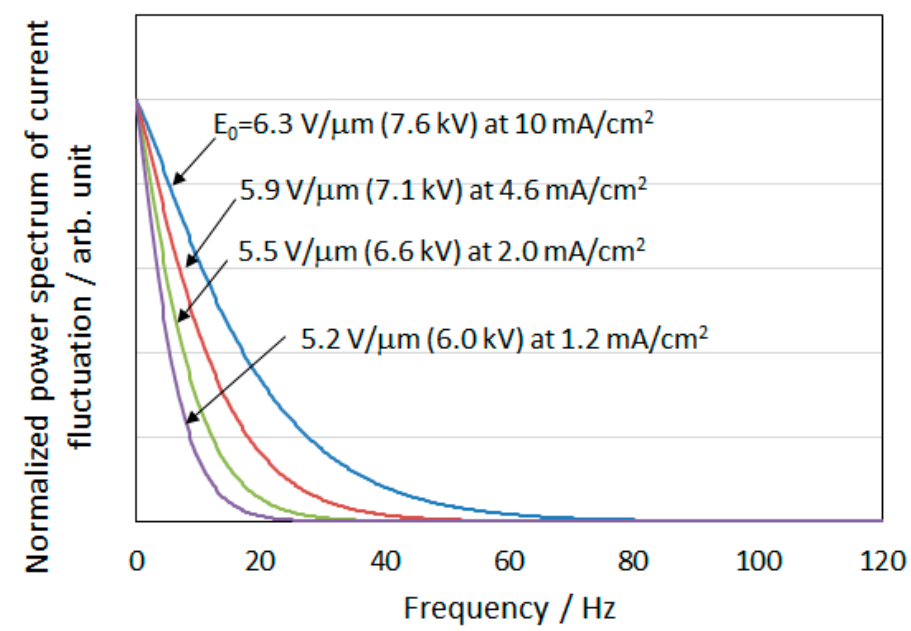

Figure 6. Dependence of power spectrum by simulation on the applied voltage. $E_{0}$ indicates the original field supplied between the cathode and anode electrodes.

FE using metallic SWCNTs was thus successfully modeled as a phenomenon based on inelastic electron tunneling using metallic SWCNTs, as shown in Figure 6. Although many researchers have reported that field emitters realize Fowler-Nordheim tunneling with elastic electrical conductivity, no demonstrative experiments have been presented to the best of our knowledge. The current paper presents the first successful development of an inelastic-electron-tunneling model with the WKB approximation for metallic SWCNTs based on the evaluation of FE properties. The FE current from field emitters, for example, SWCNTs, fluctuates, and the $I-V$ characteristics of FEs were examined by considering the power spectrum at $\omega=0$ in Equation (13), which represents the magnitude of the current due to the electrons passing through a potential barrier from the SWCNTs, in accordance with Equation (13), constructed by the inelastic-electron-tunneling model.

\section{Conclusions}

We consider that field emitters can be used as an electron-emission source in CL devices. The field emitters that are used in CL devices, including lighting devices employing visible rays, $\mathrm{X}$-rays, and ultraviolet radiation, suffer from the problem that the radiation from the CL device has a fluctuating output that depends on the electrical properties of the field emitters in time and space. In this study, we analyzed the fluctuation of the output when field emitters are used in a CL device, where we employed metallic SWCNTs as field emitters. The homogeneous dispersion of metallic SWCNTs with high crystallinity is one of the essential design requirements in the fabrication of the cathode in CL devices in order to obtain high output stability. However, it was difficult for the highly crystalline SWCNTs to control homogeneous dispersion into a mixture to make an FE cathode device. We have realized the homogeneous dispersion of metallic SWCNTs with high crystallinity for the first time by an agitation process with the ITO precursor solution, butyl acetate added, as is required to control the viscosity of the solution, and EC as a surfactant at a weight ratio of 1:8:600:10. It was difficult to disperse highly crystalline carbon nanotubes homogeneously, because no surfactant can combine to a surface of a nanotube with high crystallization. In this study, we succeeded to disperse the solution with the designed composition by controllable wet dispersion process. The metallic highly crystalline SWCNTs were employed as field emitters to analyze the fluctuation in the FE of conventional materials.

We succeeded in constructing a physical mechanism to examine FE fluctuation. Fluctuation of the FE current was observed using the metallic SWCNTs as field emitters, which was compared with the theoretical spectra calculated using a current-fluctuation theory that incorporates a realistic power spectrum of the current fluctuation in the Fowler-Nordheim tunneling regime. Good agreement was seen between the observed and the theoretically calculated spectra of the FE current obtained using 
the SWCNTs. We consider that inelastic electron tunneling causes the current fluctuation in the FE. The mechanism behind this can be explained by the superposition of the high frequency components of FE current in time and space. We also succeeded in developing a theoretical model of the flow of electrons (current) by considering the fluctuation of the tunnel current due to electrons passing through a potential barrier in the FE regime. The tunneling matrix based on the potential barrier that was obtained using the WKB approximation method is an important parameter for developing an inelastic-electron-tunneling model, and was used to obtain the power spectrum in this study. The FE model was obtained by combining the Fowler-Nordheim tunneling model with the power spectrum obtained using the tunneling matrix.

From the above results, the use of metallic SWCNTs with high crystallinity as field emitters was found to generate a fluctuating FE current, where the fluctuation originated from inelastic electron tunneling.

We have provided a brief explanation of the FE current fluctuation of metallic SWCNTs in terms of their electrical conductivity, and described the development of a conduction model for electrons that passed through a potential barrier. Highly crystalline SWCNTs are expected to be used as field emitters with stable FE.

Acknowledgments: This work was supported in part by DOWA Holdings Co. Ltd., Tokyo, Japan. The authors would like to thank the co-researchers from DOWA for the discussions and suggestions, and are grateful for the technical instructions provided during the device-assembly process and the assembly work. This study was conducted as a project consigned by the New Energy and Industrial Technology Development Organization and supported by JSPS KAKENHI Grant Number 26220104, and the authors would like to express their sincere gratitude for the guidance received.

Author Contributions: The two authors equally contributed to the paper.

Conflicts of Interest: The authors declare no conflict of interest.

\section{References}

1. Saito, Y.; Uemura, S. Field emission from carbon nanotubes and its application to electron sources. Carbon 2000, 38, 169-182. [CrossRef]

2. Saito, Y. Carbon nanotube field emitter. J. Nanosci. Nanotechnol. 2003, 3, 39-50. [CrossRef] [PubMed]

3. Kita, S.; Sakai, Y.; Endo, T.; Sugimoto, W.; Goto, H. Stabilization of field electron emission from carbon nanofibers using ballast resistance. J. Vac. Sci. Technol. B 2012, 30, 31801. [CrossRef]

4. Qian, L.; Wang, Y.-Q.; Liu, L.; Fan, S.-S. Nanoscale field emission in inert gas under atmospheric pressure. J. Vac. Sci. Technol. B 2010, 28, 562-566. [CrossRef]

5. Chichibu, S.; Sota, T.; Wada, K.; Nakamura, S. Exciton localization in InGaN quantum well devices. J. Vac. Sci. Technol. B 1998, 16, 2204-2214. [CrossRef]

6. He, G.; Chikyow, T.; Chen, X.-S.; Chen, H.-S.; Liu, J.-W.; Sun, Z.-Q. Cathodoluminescence and field emission from $\mathrm{GaN} / \mathrm{MgAl}_{2} \mathrm{O}_{4}$ grown by metalorganic chemical vapor deposition: Substrate-orientation dependence. J. Mater. Chem. C 2013, 1, 238-245. [CrossRef]

7. Yuan, X.; Zhu, W.; Zhang, Y.; Xu, N.; Yan, Y.; Wu, J.; Shen, Y.; Chen, J.; She, J.; Deng, S. A fully-sealed carbon-nanotube cold-cathode terahertz gyrotron. Sci. Rep. 2016, 6, 32936. [CrossRef] [PubMed]

8. Burtsev, A.A.; Grigorev, Y.A.; Navrotsky, I.A.; Rogovin, V.I.; Sakhadzhi, G.V.; Shumikhin, K.V. Experimental investigation of electron guns for THz microwave vacuum amplifiers. Tech. Phys. Lett. 2016, 42, 543-545. [CrossRef]

9. Gamzina, D.; Himes, L.G.; Barchfeld, R.; Zheng, Y.; Popovic, B.K.; Paoloni, C.; Choi, E.-M.; Luhmann, N.C., Jr. Nano-CNC machining of sub-THz vacuum electron devices. IEEE Trans. Electron Devices 2016, 63, 4067-4073. [CrossRef]

10. Zhao, J.; Yin, H.; Zhang, L.; Shu, G.; He, W.; Zhang, J.; Zhang, Q. Influence of the electrode gap separation on the pseudospark-sourced electron beam generation. Phys. Plasmas 2016, 23, 73116. [CrossRef]

11. Heeger, A.J.; Parker, I.D.; Yang, Y. Carrier injection into semiconducting polymers: Fowler-Nordheim field-emission tunneling. Synth. Met. 1994, 67, 23-29. [CrossRef] 
12. Fowler, R.H.; Nordheim, L. Electron emission in intense electric fields. Proc. R. Soc. Lond. Ser. A 1928, 119, 173-181. [CrossRef]

13. Shimoi, N.; Adriana, L.E.; Tanaka, Y.; Tohji, K. Properties of a field emission lighting plane employing highly crystalline single-walled carbon nanotubes fabricated by simple processes. Carbon 2013, 65, 228-235. [CrossRef]

14. Garrido, S.B.; Shimoi, N.; Abe, D.; Hojo, T.; Tanaka, Y.; Tohji, K. Planar light source using a phosphor screen with single-walled carbon nanotubes as field emitters. Rev. Sci. Instrum. 2014, 85, 104704. [CrossRef] [PubMed]

15. Iijima, S. Helical microtubules of graphitic carbon. Nature 1991, 354, 56-58. [CrossRef]

16. Ebbesen, T.W.; Lezec, H.J.; Hiura, H.; Bennett, J.W.; Ghaemi, H.F.; Thio, T. Electrical conductivity of individual carbon nanotubes. Nature 1997, 382, 54-56. [CrossRef]

17. Wildoerm, J.W.G.; Venema, L.C.; Rinzler, A.G.; Smalley, R.E.; Dekker, C. Electronic structure of atomically resolved carbon nanotubes. Nature 1998, 391, 59-62. [CrossRef]

18. Odom, T.W.; Huang, J.L.; Kim, P.; Lieber, C.M. Atomic structure and electronic properties of single-walled carbon nanotubes. Nature 1998, 391, 62-64. [CrossRef]

19. Treacy, M.M.J.; Ebbesen, T.W.; Gibson, J.M. Exceptionally high Young's modulus observed for individual carbon nanotubes. Nature 1996, 381, 678-680. [CrossRef]

20. Dai, H.; Hafner, J.H.; Rinzler, A.G.; Colbert, D.T.; Smalley, R.E. Nanotubes as nanoprobes in scanning probe microscopy. Nature 1996, 384, 147-150. [CrossRef]

21. Dillon, A.C.; Jones, K.M.; Bekkedahl, T.A.; Kiang, C.H.; Bethune, D.S.; Haben, M.J. Storage of hydrogen in single-walled carbon nanotubes. Nature 1997, 386, 377-380. [CrossRef]

22. Niu, C.; Sichel, E.K.; Hoch, R.; Moy, D.; Tennent, H. High power electrochemical capacitors based on carbon nanotube electrodes. Appl. Phys. Lett. 1997, 70, 1480-1482. [CrossRef]

23. Shimoi, N.; Abe, D.; Matsumoto, K.; Sato, Y.; Tohji, K. Low-power-consumption flat-panel light-emitting device driven by field-emission electron source using high-crystalline single-walled carbon nanotube. Jpn. J. Appl. Phys. 2017, 56, 07GE01. [CrossRef]

24. Iwata, S.; Sato, Y.; Nakai, K.; Ogura, S.; Okano, T.; Namura, M.; Kasuya, A.; Tohji, K.; Fukutani, K. Novel method to evaluate the carbon network of single-walled carbon nanotubes by hydrogen physisorption. J. Phys. Chem. C Lett. 2007, 111, 14937-14941. [CrossRef]

25. Shimoi, N.; Abe, D.; Tanaka, Y.; Tohji, K. A stand-alone flat-plane lighting device in a diode structure employing highly crystalline SWCNTs as field emitters. Diamond Relat. Mater. 2016, 65, 152-157. [CrossRef]

26. Spindt, C.A.; Brodie, I.; Humphrey, L.; Westerberg, E.R. Physical properties of thin-film field emission cathodes with molybdenum cones. J. Appl. Phys. 1976, 47, 5248-5263. [CrossRef]

27. Laks, B.; Mills, D.L. Photon emission from slightly roughened tunnel junctions. Phys. Rev. B 1979, $20,4962$. [CrossRef]

28. Watanabe, J.; Uehara, Y.; Ushioda, S. Multiple-scattering effect of surface-plasmon polaritons in light emission from tunnel junction. Phys. Rev. B 1995, 52, 2860-2867. [CrossRef]

29. Uehara, Y.; Watanabe, J.; Fujikawa, S.; Ushioda, S. Light-emission mechanism of Si-MOS tunnel junctions. Phys. Rev. B 1995, 51, 2229-2238. [CrossRef]

30. Hone, D.; Muhlschlegel, B.; Scalapino, D.J. Theory of light emission from small particle tunnel junctions. Appl. Phys. Lett. 1998, 33, 203. [CrossRef]

31. Shimoi, N.; Tanaka, S.-I. Numerical analysis of electron emission site distribution of carbon nanofibers for field emission properties. ACS Appl. Mater. Interfaces 2012, 5, 768-773. [CrossRef] [PubMed]

32. Shimoi, N.; Tanaka, S.-I. Numerically optimized bundle size and distribution of carbon nanofibers for a field emitter. Carbon 2010, 48, 905-911. [CrossRef]

33. Bandaru, P.R. Electrical properties and applications of carbon nanotube structure. J. Nanosci. Nanotechnol. 2007, 7, 1239-1267. [CrossRef] [PubMed]

(C) 2017 by the authors. Licensee MDPI, Basel, Switzerland. This article is an open access article distributed under the terms and conditions of the Creative Commons Attribution (CC BY) license (http:/ / creativecommons.org/licenses/by/4.0/). 